\title{
Bethanechol-Induced Water Intake in Rats: Possible Mechanisms of Induction ${ }^{1}$
}

\author{
MELVIN J. FREGLY, DIANNE C. KIKTA AND JOHN E. GREENLEAF ${ }^{2}$ \\ Department of Physiology, University of Florida, College of Medicine, Gainesville, FL 32610
}

Received 10 April, 1982

\begin{abstract}
FREGLY, M. J., D. C. KIKTA AND J. E. GREENLEAF. Bethanechol-induced water intake in rats: Possible mechanisms of induction. PHARMAC. BIOCHEM. BEHAV. 17(4) 727-732, 1982.-Acute administration of the parasympathomimetic agent, bethanechol, at $2,4,8$ and $12 \mathrm{mg} / \mathrm{kg}$ body $\mathrm{wt}$, IP, induced drinking and increased urine output of rats in a dose-dependent fashion. The first significant increases in both water intake and urine output above that of controls occurred when $4 \mathrm{mg} / \mathrm{kg}$ was administered. The drinking and increased urine output in response to administration of $8 \mathrm{mg}$ bethanechol $/ \mathrm{kg}$ was inhibited by atropine sulfate $(3$ and $6 \mathrm{mg} / \mathrm{kg}$, IP). In addition, the $\beta$-adrenergic antagonist, propranolol $(6 \mathrm{mg} / \mathrm{kg}$, IP, administered $30 \mathrm{~min}$ prior to treatment with bethanechol), inhibited bethanechol $(8 \mathrm{mg} / \mathrm{kg}$, IP)-induced drinking. Urine output, however, was unaffected by propranolol. Further, the angiotensin I converting enzyme inhibitor, captopril, inhibited significantly the drinking response, but not the increased urine output, accompanying administration of bethanechol $(8 \mathrm{mg} / \mathrm{kg})$. The effect of bethanechol and the $\beta$-adrenergic agonist, isoproterenol $(25 \mu \mathrm{g} / \mathrm{kg})$ separately and in combination, on water intake was also studied. Both compounds increased water intake but they exerted no interactive effect when administered simultaneously. Administration of bethanechol $(8 \mathrm{mg} / \mathrm{kg})$ to conscious rats was also accompanied by a significant reduction in both mean blood pressure and heart rate that reached minimal levels within 10 min after treatment. Both responses had returned to control level by one hr after treatment. These results suggest that bethanechol induces drinking in rats by way of the renin-angiotensin system.
\end{abstract}

Parasympathomimetic agents Bethanechol Drinking Renin-angiotensin system

BOTH central and peripheral administration of parasympathomimetic agents is well known to initiate drinking in rats $[1-6,11]$. Of the two routes of administration, the peripheral route appears to initiate drinking less reliably [5]. Thus, the dose-response relationship between dose of pilocarpine administered and water intake during 90 minutes after treatment was not linear and actually decreased when doses higher than $3 \mathrm{mg} / \mathrm{kg}$ of body weight were administered intraperitoneally [5]. An initial objective was to find a parasympathomimetic agent that could induce drinking more reliably than pilocarpine when administered peripherally. Initial studies with peripherally administered carbachol were unsuccessfull in that increasing doses failed to induce drinking and actually resulted in the death of several rats at the highest dose used $(1 \mathrm{mg} / \mathrm{kg}$, IP). Bethanechol, another parasympathomimetic agent, was tested and found to induce dipsogensis in a dose-dependent fashion when graded doses were administered to rats. Additional studies were then conducted to assess by pharmacologic means whether the renin-angiotensin system was involved in the drinking response.

\section{METHOD}

Female rats of the Blue Spruce Farms (Sprague-Dawley) strain were used in all experiments. The rats ranged in weight from 250 to $350 \mathrm{~g}$ at the beginning of the experiments. All animals were kept in a thermoregulated $\left(26 \pm 1^{\circ} \mathrm{C}\right)$ room illuminated from 7 a.m. to 7 p.m. Purina Laboratory Chow and tap water were provided ad lib. All drinking studies were performed in a quiet, thermoregulated $\left(26 \pm 1^{\circ} \mathrm{C}\right)$ room beginning at 9:30 a.m. Water was used to dissolve all compounds administered in these studies.

Experiment I: Effect of Carbamyl- $\beta$-Methyl-Choline Chloride (Bethanechol) on Water Intake

Thirty naive female rats $(280-330 \mathrm{~g})$ were divided randomly into five equal groups. Groups 1 through 4 received bethanechol at $2,4,8$ and $12 \mathrm{mg} / \mathrm{kg}$, IP. Group 5 served as control and received the vehicle used to dissolve bethanechol. After administration of the drugs, each rat was placed in an individual stainless steel metabolism cage and given a preweighed water bottle containing distilled water [10]. Water intake and urine output were measured at hourly intervals for three hr. The three urine samples of each rat were pooled and analyzed for their sodium and potassium concentrations by flame photometry.

Analysis of the data was made by a one-way analysis of variance [13]. Comparison between individual means was made by the t-test using the pooled variance from the analysis of variance [7].

\section{Experiment 2: Effect of Atropine on Bethanechol-Induced} Water Intake

Twenty-four naive female rats (290 to $350 \mathrm{~g}$ ) were divided

'Supported by contract NCA2-OR204-101 from the National Aeronautics and Space Administration, Moffett Field, CA 94035.

2Present address: Biomedical Research Division, Ames Research Center NASA, Moffett Field, CA 94035. 
into four equal groups. Groups 1-3 received bethanechol ( 8 $\mathrm{mg} / \mathrm{kg}$, IP) while the second, and third groups also received the cholinergic receptor antagonist, atropine sulfate, at 3 and $6 \mathrm{mg} / \mathrm{kg}$, IP, respectively. The remaining group served as control and received the vehicle used to dissolve both the bethanechol and atropine. Atropine was administered $15 \mathrm{~min}$ prior to administration of bethanechol. After administration of the compounds, each rat was placed in a stainless steel metabolism cage and given a preweighed bottle of distilled water. Water intake and urine outputs were measured at hourly intervals for three hr thereafter. Urinary sodium and potassium concentrations were measured by flame photometry on the urine collected from each rat during the entire three $\mathrm{hr}$ of the experiment.

Analysis of the data was made as described in Experiment 1.

Experiment 3: Effect of Propranolol on BethanecholInduced Water Intake

The same 24 rats used in Experiment 2 were used in this experiment. One week elapsed between experiments. The rats were divided randomly into four equal groups. Group 1 received bethanechol ( $8 \mathrm{mg} / \mathrm{kg}$, IP) while the second and third groups received bethanechol in combination with 3 and $6 \mathrm{mg} \mathrm{d}, 1$-propranolol- $\mathrm{HCl} / \mathrm{kg}$, IP, respectively. Propranolol, a $\beta$-adrenoreceptor blocker, was administered $30 \mathrm{~min}$ prior to administration of bethanechol. The remainder of the experiment was carried out identically to that of Experiment 2.

Experiment 4: Effect of Captopril on Bethanechol-Induced Water Intake

The same rats used in Experiment 3 were used in this experiment. One week elapsed between experiments. The rats were divided randomly into four equal groups. Group 1 received bethanechol $(8 \mathrm{mg} / \mathrm{kg}$, IP) while the second group received captopril, an angiotensin I converting enzyme inhibitor (35 mg/kg, IP). The third group received both bethanechol and captopril while the fourth group was administered the vehicle used to dissolve both captopril and bethanechol and served as control. Both compounds were administered at the same time. The remainder of the experiment was carried out identically to that described in Experiment 2 .

Data from this study were analyzed by a two-way analysis of variance to assess the effect of treatment with each compound as well as their interaction [13].

Experiment 5: Effect of Administration of Isoproterenol and Bethanechol, Alone and Combined, on Water Intake

Twenty four naive rats (235 to $275 \mathrm{~g}$ ) were divided randomly into four equal groups. Group 1 was administered d,l-isoproterenol $\mathrm{HCl}$, a $\beta$-adrenergic agonist $(25 \mu \mathrm{g} / \mathrm{kg}, \mathrm{SC})$ while Group 2 received bethanechol $(8 \mathrm{mg} / \mathrm{kg}$, IP). Group 3 received both compounds simultaneously while Group 4 received the vehicle used to dissolve both compounds. The remainder of the experiment was carried out as described in Experiment 2 except that urine output and urinary excretion of sodium and potassium were not measured.

Data from this study were analyzed by a two way analysis of variance as described in Experiment 4.

Experiment 6: Effect of Bethanechol on Blood Pressure and Heart Rate of Conscious Rats

Six female rats ( 260 to $290 \mathrm{~g}$ ) were used. Each rat had an indwelling carotid arterial cannula that had been in place for several weeks prior to the study. Blood pressure was monitored continuously by attaching the cannula to a Statham $\mathrm{P} 23 \mathrm{Db}$ pressure transducer which was coupled to a Grass polygraph. The conscious, unrestrained rats were kept in their cages throughout the experiment. Heparinized saline $(100 \mathrm{U} / \mathrm{ml})$ was used in all cannulae to keep them patent.

On the day of the study, two groups of rats ( 3 each) were used. After an initial control period lasting 30 minutes, either saline or bethanechol $(8 \mathrm{mg} / \mathrm{kg})$ was administered IP. Both blood pressure and heart rate were measured at the end of the control period and 1, 3 and 5 min after treatment with bethanechol and at $5 \mathrm{~min}$ intervals thereafter for $55 \mathrm{~min}$.

RESULTS

\section{Experiment I}

Administration of graded doses of bethanechol increased water intake during the first hr in a dose-dependent fashion up to the highest dose administered $(12 \mathrm{mg} / \mathrm{kg}$, Table 1$)$. The first significant $(p<0.05)$ increase in water intake occurred when a dose of $4 \mathrm{mg} / \mathrm{kg}$ was administered. The smaller water intake accompanying administration of the highest dose of bethanechol was apparently related to toxicity. During the second hr when the toxic manifestation had subsided, water intake more than doubled that observed during the first hr. A further increase in the water intake of this group, which was considerably in excess of that observed for any of the other treated groups, occurred during the third hr of the experiment.

Urine output also increased in a dose-dependent fashion during the three hr of the experiment (Table 1). Both urinary sodium and potassium excretions also increased with increases in the dose of bethanechol administered. The increases in urinary sodium and potassium excretion occurred in roughly similar amounts since the urinary $\mathrm{Na} / \mathrm{K}$ ratio of any one of the treated groups failed to differ significantly from that of the control group.

\section{Experiment 2}

Administration of atropine sulfate at either 3 or $6 \mathrm{mg} / \mathrm{kg}$, IP, inhibited the increased water intake induced by bethanechol (Table 2). Both doses appeared to be equally potent in this regard. The inhibitory effect of atropine persisted throughout the three hr of the experiment. In addition, the increased urinary output induced by bethanechol was prevented by administration of either dose of atropine as was the increased urinary sodium and potassium output.

\section{Experiment 3}

Administration of the $\beta$-adrenergic antagonist, $\mathrm{d}, \mathrm{l}$ propranolol, inhibited the increased water intake induced by bethanechol (Table 3 ). The higher dose of propranolol $(6$ $\mathrm{mg} / \mathrm{kg}$ ) inhibited water intake to a greater extent than the lower dose $(3 \mathrm{mg} / \mathrm{kg})$. The effect of the higher dose of propranolol persisted throughout the three hr of the experiment. Administration of propranolol in combination with bethanechol failed to affect the increased urine output and the increased urinary sodium and potassium excretion rates induced by bethanechol. Urinary $\mathrm{Na} / \mathrm{K}$ ratios of all treated groups were significantly $(p<0.01)$ greater than that of controls. 
TABLE 1

EFFECT OF CARBAMYL $\beta$-METHYL-CHOLINE (BETHANECHOL) ON WATER INTAKE, URINE OUTPUT, AND URINE ELECTROLYTE EXCRETION BY FEMALE RATS

\begin{tabular}{|c|c|c|c|c|c|c|c|c|c|c|c|}
\hline $\begin{array}{c}\text { Experimental } \\
\text { Group }\end{array}$ & $\begin{array}{c}\text { Number } \\
\text { of } \\
\text { Rats }\end{array}$ & $\begin{array}{l}\text { Mean } \\
\text { Body } \\
\text { wt. (g) }\end{array}$ & \multicolumn{3}{|c|}{$\begin{array}{c}\text { Cumulative } \\
\text { Water Intake } \\
\text { (ml/kg body wt.) } \\
\text { during }\end{array}$} & \multicolumn{3}{|c|}{$\begin{array}{c}\text { Cumulative } \\
\text { Urine Output } \\
\text { (ml/kg body wt.) } \\
\text { during }\end{array}$} & $\begin{array}{c}\text { Urinary } \\
\mathrm{Na} \\
\text { Excretion } \\
(\mathrm{mEq} / \mathrm{kg} / 3 \mathrm{hr})\end{array}$ & $\begin{array}{c}\text { Urinary } \\
\mathrm{K} \\
\text { Excretion } \\
(\mathrm{mEq} / \mathrm{kg} / 3 \mathrm{hr})\end{array}$ & $\begin{array}{c}\text { Urinary } \\
\text { Na/K } \\
\text { Ratio }\end{array}$ \\
\hline \multirow[t]{3}{*}{ Control } & 6 & 325 & 1.8 & 2.2 & 2.8 & 1.2 & 1.2 & 1.2 & 0.20 & 0.12 & 1.66 \\
\hline & & \pm & \pm & \pm & \pm & \pm & \pm & \pm & \pm & \pm & \pm \\
\hline & & $8^{*}$ & 0.3 & 0.2 & 0.2 & 0.5 & 0.5 & 0.5 & 0.05 & 0.01 & 0.44 \\
\hline Bethanechol & 6 & 322 & 2.3 & 2.8 & 3.5 & 1.9 & 2.7 & 3.6 & 0.53 & 0.18 & 2.81 \\
\hline$(2 \mathrm{mg} / \mathrm{kg}$ & & \pm & \pm & \pm & \pm & \pm & \pm & \pm & \pm & \pm & \pm \\
\hline IP) & & 5 & 1.2 & 1.2 & 1.2 & 0.7 & 1.0 & 1.1 & 0.16 & 0.04 & 0.38 \\
\hline Bethanechol & 6 & 308 & 5.3 & 5.8 & 6.4 & 3.6 & 4.3 & 5.8 & 0.49 & 0.23 & 2.25 \\
\hline$(4 \mathrm{mg} / \mathrm{kg}$ & & \pm & \pm & \pm & \pm & \pm & \pm & \pm & \pm & \pm & \pm \\
\hline IP) & & 11 & $0.9+$ & $0.9 \ddagger$ & $0.9 \ddagger$ & 1.0 & 1.0 & 0.9 t & $0.08 \mp$ & $0.03 \ddagger$ & 0.36 \\
\hline Bethanechol & 6 & 310 & 9.8 & 11.1 & 11.7 & 4.8 & 5.4 & 8.6 & 0.77 & 0.29 & 2.72 \\
\hline$(8 \mathrm{mg} / \mathrm{kg}$ & & \pm & \pm & \pm & \pm & \pm & \pm & \pm & \pm & \pm & \pm \\
\hline IP) & & 6 & 1.8 & $2.6 \div$ & $2.6+$ & 2.6 & $0.8 \ddagger$ & $1.1 \div$ & 0.17 丰 & $0.01 \ddagger$ & 0.48 \\
\hline Bethanechol & 6 & 322 & 5.1 & 12.1 & 14.2 & 7.5 & 8.5 & 11.6 & 0.75 & 0.42 & 2.12 \\
\hline$(12 \mathrm{mg} / \mathrm{kg}$ & & \pm & \pm & \pm & \pm & \pm & \pm & \pm & \pm & \pm & \pm \\
\hline IP) & & 14 & 2.8 & $2.9+$ & $3.0 \ddagger$ & $1.6 \neq$ & $1.4 \ddagger$ & $2.7 \ddagger$ & $0.12 \ddagger$ & $0.11^{\dagger}$ & 0.29 \\
\hline
\end{tabular}

* One standard error of mean.

+ Significantly different from Control $(p<0.05)$.

$\ddagger$ Significantly different from Control $(p<0.01)$.

TABLE 2

EFFECT OF CARBAMYL $\beta$-METHYL-CHOLINE (BETHANECHOL) AND ATROPINE SULFATE ALONE AND IN COMBINATION, ON WATER INTAKE, URINE OUTPUT AND URINE ELECTROLYTE EXCRETION BY FEMALE RATS

\begin{tabular}{|c|c|c|c|c|c|c|c|c|c|c|c|}
\hline \multirow{2}{*}{$\begin{array}{l}\text { Experimental } \\
\text { Group }\end{array}$} & \multirow{2}{*}{$\begin{array}{c}\text { Number } \\
\text { of } \\
\text { Rats }\end{array}$} & \multirow{2}{*}{$\begin{array}{c}\text { Mean } \\
\text { Body } \\
\text { wt. (g) }\end{array}$} & \multicolumn{3}{|c|}{$\begin{array}{c}\text { Cumulative } \\
\text { Water Intake } \\
\text { (ml/kg body wt.) } \\
\text { during }\end{array}$} & \multicolumn{3}{|c|}{$\begin{array}{l}\text { Cumulative } \\
\text { Urine Output } \\
\text { (ml/kg body wt.) } \\
\text { during }\end{array}$} & \multirow{2}{*}{$\begin{array}{c}\text { Urinary } \\
\mathrm{Na} \\
\text { Excretion } \\
(\mathrm{mEq} / \mathrm{kg} / 3 \mathrm{hr})\end{array}$} & \multirow{2}{*}{$\begin{array}{c}\text { Urinary } \\
\mathrm{K} \\
\text { Excretion } \\
(\mathrm{mEq} / \mathrm{kg} / 3 \mathrm{hr})\end{array}$} & \multirow{2}{*}{$\begin{array}{c}\text { Urinary } \\
\mathrm{Na} / \mathrm{K} \\
\mathrm{Ratio}\end{array}$} \\
\hline & & & 1 & 2 & $3 \mathrm{hr}$ & 1 & 2 & $3 \mathrm{hr}$ & & & \\
\hline Control & 6 & $\begin{array}{c}329 \\
\pm \\
9^{*}\end{array}$ & $\begin{array}{c}1.4 \\
\pm \\
0.4\end{array}$ & $\begin{array}{l}1.7 \\
\pm \\
0.5\end{array}$ & $\begin{array}{c}2.2 \\
\pm \\
0.5\end{array}$ & $\begin{array}{r}2.0 \\
\pm \\
0.9\end{array}$ & $\begin{array}{r}2.9 \\
\pm \\
1.1\end{array}$ & $\begin{array}{r}3.3 \\
\pm \\
1.2\end{array}$ & $\begin{array}{r}0.32 \\
\pm \\
0.04\end{array}$ & $\begin{array}{r}0.29 \\
\pm \\
0.09\end{array}$ & $\begin{array}{c}1.24 \\
\pm \\
0.24\end{array}$ \\
\hline $\begin{array}{l}\text { Bethanechol } \\
(8 \mathrm{mg} / \mathrm{kg} \text {, } \\
\text { IP) }\end{array}$ & 6 & $\begin{array}{c}322 \\
\pm \\
11\end{array}$ & $\begin{array}{l}9.4 \\
\pm \\
2.1+\end{array}$ & $\begin{array}{l}11.7 \\
\pm \\
1.4 \ddagger\end{array}$ & $\begin{array}{l}12.4 \\
\pm \\
1.4 \div\end{array}$ & $\begin{array}{l}4.7 \\
\pm \\
0.7+\end{array}$ & $\begin{array}{l}5.8 \\
\pm \\
0.9+\end{array}$ & $\begin{array}{l}8.3 \\
\pm \\
0.9 \ddagger\end{array}$ & $\begin{array}{c}0.77 \\
\pm \\
0.08\end{array}$ & $\begin{array}{c}0.64 \\
\pm \\
0.07^{+}\end{array}$ & $\begin{array}{c}1.26 \\
\pm \\
0.18\end{array}$ \\
\hline $\begin{array}{l}\text { Bethanechol } \\
+ \text { Atropine } \\
(3 \mathrm{mg} / \mathrm{kg} \\
\text { IP) \# }\end{array}$ & 6 & $\begin{array}{c}324 \\
\pm \\
15\end{array}$ & $\begin{array}{l}2.9 \\
\pm \\
1.39\end{array}$ & $\begin{array}{c}2.9 \\
\pm \\
1.39\end{array}$ & $\begin{array}{l}3.4 \\
\pm \\
1.44\end{array}$ & $\begin{array}{c}0.0 \\
\pm \\
0.0+9\end{array}$ & $\begin{array}{l}0.0 \\
\pm \\
0.0 \pm \uparrow\end{array}$ & $\begin{array}{c}0.9 \\
\pm \\
0.99\end{array}$ & $\begin{array}{c}0.00 \\
\pm \\
0.00 \pm \uparrow\end{array}$ & $\begin{array}{l}0.00 \\
\pm \\
0.00 \ddagger \uparrow\end{array}$ & $\begin{array}{c}0.00 \\
\pm \\
0.00\end{array}$ \\
\hline $\begin{array}{l}\text { Bethanechol } \\
+ \text { Atropine } \\
\text { (6 mg/kg, } \\
\text { IP) \# }\end{array}$ & 6 & $\begin{array}{c}316 \\
\pm \\
6\end{array}$ & $\begin{array}{c}2.0 \\
\pm \\
0.49\end{array}$ & $\begin{array}{l}2.2 \\
\pm \\
0.5\end{array}$ & $\begin{array}{l}3.0 \\
\pm \\
0.59\end{array}$ & $\begin{array}{l}0.9 \\
\pm \\
0.7\end{array}$ & $\begin{array}{l}2.1 \\
\pm \\
1.18\end{array}$ & $\begin{array}{c}2.1 \\
\pm \\
1.29\end{array}$ & $\begin{array}{r}0.27 \\
\pm \\
0.06\end{array}$ & $\begin{array}{r}0.31 \\
\pm \\
0.05\end{array}$ & $\begin{array}{c}0.85 \\
\pm \\
0.04\end{array}$ \\
\hline
\end{tabular}

*One standard error of mean.

† Significantly different from Control $(p<0.05)$.

$\ddagger$ Significantly different from Control $(p<0.01)$.

$\S$ Significantly different from Bethanechol $(p<0.05)$.

TSignificantly different from Bethanechol $(p<0.01)$.

\#Atropine sulfate given 15 minutes before Bethanechol. 
TABLE 3

EFFECT OF CARBAMYL $\beta$-METHYL-CHOLINE (BETHANECHOL) AND PROPRANOLOL, ALONE AND IN COMBINATION, ON WATER INTAKE, URINE OUTPUT AND URINE ELECTROLYTE EXCRETION BY FEMALE RATS

\begin{tabular}{|c|c|c|c|c|c|c|c|c|c|c|c|}
\hline \multirow{2}{*}{$\begin{array}{l}\text { Experimental } \\
\text { Group }\end{array}$} & \multirow{2}{*}{$\begin{array}{c}\text { Number } \\
\text { of } \\
\text { Rats }\end{array}$} & \multirow{2}{*}{$\begin{array}{l}\text { Mean } \\
\text { Body } \\
\text { wt. (g) }\end{array}$} & \multicolumn{3}{|c|}{$\begin{array}{c}\text { Cumulative } \\
\text { Water Intake } \\
\text { (ml/kg body wt.) } \\
\text { during }\end{array}$} & \multicolumn{3}{|c|}{$\begin{array}{l}\text { Cumulative } \\
\text { Urine Output } \\
\text { (ml/kg body wt.) } \\
\text { during }\end{array}$} & \multirow{2}{*}{$\begin{array}{c}\text { Urinary } \\
\text { Na } \\
\text { Excretion } \\
\text { (mEq/kg/3 hr) }\end{array}$} & \multirow{2}{*}{$\begin{array}{c}\text { Urinary } \\
\mathrm{K} \\
\text { Excretion } \\
(\mathrm{mEq} / \mathrm{kg} / 3 \mathrm{hr})\end{array}$} & \multirow{2}{*}{$\begin{array}{c}\text { Urinary } \\
\mathrm{Na} / \mathrm{K} \\
\text { Ratio }\end{array}$} \\
\hline & & & 1 & 2 & $3 \mathrm{hr}$ & 1 & 2 & $3 \mathrm{hr}$ & & & \\
\hline \multirow[t]{3}{*}{ Control } & \multirow[t]{3}{*}{6} & 311 & 2.5 & 3.0 & 3.6 & 1.0 & 1.8 & 3.3 & 0.22 & 0.32 & 0.67 \\
\hline & & \pm & \pm & \pm & \pm & \pm & \pm & \pm & \pm & \pm & \pm \\
\hline & & $11^{*}$ & 0.9 & 0.9 & 0.9 & 1.0 & 1.5 & 1.4 & 0.05 & 0.05 & 0.09 \\
\hline \multirow{3}{*}{$\begin{array}{l}\text { Bethanechol } \\
(8 \mathrm{mg} / \mathrm{kg} \text {, } \\
\text { IP) }\end{array}$} & \multirow[t]{3}{*}{6} & 328 & 10.3 & 11.8 & 12.1 & 5.7 & 6.8 & 8.8 & 0.79 & 0.59 & 1.86 \\
\hline & & \pm & \pm & \pm & \pm & \pm & \pm & \pm & \pm & \pm & \pm \\
\hline & & 11 & $2.5^{\dagger}$ & $1.9 \ddagger$ & $1.9 \ddagger$ & $1.3 \frac{1}{7}$ & $1.5 \ddagger$ & $1.8 \ddagger$ & $0.17 \ddagger$ & $0.13 \div$ & $0.54 \ddagger$ \\
\hline \multirow{3}{*}{$\begin{array}{l}\text { Bethanechol } \\
+ \text { Propranolol } \\
\text { (3 mg/kg, } \\
\text { IP) }\end{array}$} & \multirow[t]{3}{*}{6} & 325 & 5.2 & 8.1 & 9.6 & 6.8 & 7.7 & 9.3 & 0.78 & 0.56 & 1.56 \\
\hline & & \pm & \pm & \pm & \pm & \pm & \pm & \pm & \pm & \pm & \pm \\
\hline & & 15 & 3.2 & 2.9 & 2.9 & 1.5 & 1.6 & 1.7 & 0.13 & $0.11 \frac{4}{4}$ & $0.27 \ddagger$ \\
\hline \multirow{3}{*}{$\begin{array}{l}\text { Bethanechol } \\
+ \text { Propranolol } \\
(6 \mathrm{mg} / \mathrm{kg} \\
\text { IP) }\end{array}$} & \multirow[t]{3}{*}{6} & 316 & 2.0 & 2.2 & 3.0 & 4.4 & 6.1 & 2.1 & 0.83 & 0.58 & 1.48 \\
\hline & & \pm & \pm & \pm & \pm & \pm & \pm & \pm & \pm & \pm & \pm \\
\hline & & 5 & $0.2 \S$ & 0.68 & $0.6 \$$ & 1.1 & 1.4 & 1.2 & $0.13 \ddagger$ & 0.12 & $0.08 \ddagger$ \\
\hline
\end{tabular}

*One standard error of mean.

$\uparrow$ Significantly different from Control $(p<0.05)$.

$\ddagger$ Significantly different from Control $(p<0.01)$.

$\$$ Significantly different from Bethanechol $(p<0.05)$.

TPropranolol given 30 minutes before Bethanechol.

TABLE 4

EFFECT OF CARBAMYL $\beta$-METHYL-CHOLINE (BETHANECHOL) AND CAPTOPRIL, ALONE AND IN COMBINATION, ON WATER INTAKE, URINE OUTPUT AND URINE ELECTROLYTE EXCRETION BY FEMALE RATS

\begin{tabular}{|c|c|c|c|c|c|c|c|c|c|c|c|}
\hline \multirow{2}{*}{$\begin{array}{l}\text { Experimental } \\
\text { Group }\end{array}$} & \multirow{2}{*}{$\begin{array}{c}\text { Number } \\
\text { of } \\
\text { Rats }\end{array}$} & \multirow{2}{*}{$\begin{array}{l}\text { Mean } \\
\text { Body } \\
\text { wt. (g) }\end{array}$} & \multicolumn{3}{|c|}{$\begin{array}{c}\text { Cumulative } \\
\text { Water Intake } \\
\text { (ml/kg body wt.) } \\
\text { during }\end{array}$} & \multicolumn{3}{|c|}{$\begin{array}{l}\text { Cumulative } \\
\text { Urine Output } \\
\text { (ml/kg body wt.) } \\
\text { during }\end{array}$} & \multirow{2}{*}{$\begin{array}{c}\text { Urinary } \\
\mathrm{Na} \\
\text { Excretion } \\
(\mathrm{mEq} / \mathrm{kg} / 3 \mathrm{hr})\end{array}$} & \multirow{2}{*}{$\begin{array}{c}\text { Urinary } \\
\mathrm{K} \\
\text { Excretion } \\
(\mathrm{mEq} / \mathrm{kg} / 3 \mathrm{hr})\end{array}$} & \multirow{2}{*}{$\begin{array}{c}\text { Urinary } \\
\text { Na/K } \\
\text { Ratio }\end{array}$} \\
\hline & & & 1 & 2 & $3 \mathrm{hr}$ & 1 & 2 & $3 \mathrm{hr}$ & & & \\
\hline Control & 6 & $\begin{array}{l}305 \\
\pm \\
4^{*}\end{array}$ & $\begin{array}{l}1.8 \\
\pm \\
0.4\end{array}$ & $\begin{array}{l}2.8 \\
\pm \\
0.6\end{array}$ & $\begin{array}{l}3.2 \\
\pm \\
0.6\end{array}$ & $\begin{array}{l}0.3 \\
\pm \\
0.2\end{array}$ & $\begin{array}{l}0.3 \\
\pm \\
0.2\end{array}$ & $\begin{array}{l}1.1 \\
\pm \\
0.5\end{array}$ & $\begin{array}{l}0.27 \\
\pm \\
0.03\end{array}$ & $\begin{array}{l}0.16 \\
\pm \\
0.01\end{array}$ & $\begin{array}{l}1.65 \\
\pm \\
0.06\end{array}$ \\
\hline $\begin{array}{l}\text { Bethanechol } \\
(8 \mathrm{mg} / \mathrm{kg} \text {, } \\
\text { IP) }\end{array}$ & 6 & $\begin{array}{c}322 \\
\pm \\
4\end{array}$ & $\begin{array}{l}9.9 \\
\pm \\
2.7\end{array}$ & $\begin{array}{l}10.5 \\
\pm \\
2.7\end{array}$ & $\begin{array}{l}11.1 \\
\pm \\
2.7\end{array}$ & $\begin{array}{l}3.7 \\
\pm \\
0.9\end{array}$ & $\begin{array}{l}5.0 \\
\pm \\
0.8\end{array}$ & $\begin{array}{l}7.2 \\
\pm \\
1.6\end{array}$ & $\begin{array}{l}0.66 \\
\pm \\
0.04\end{array}$ & $\begin{array}{l}0.66 \\
\pm \\
0.04\end{array}$ & $\begin{array}{l}1.01 \\
\pm \\
0.05\end{array}$ \\
\hline $\begin{array}{l}\text { Captopril } \\
(35 \mathrm{mg} / \mathrm{kg} \text {. } \\
\text { IP) }\end{array}$ & 6 & $\begin{array}{c}321 \\
\pm \\
6\end{array}$ & $\begin{array}{l}1.3 \\
\pm \\
0.5\end{array}$ & $\begin{array}{l}2.9 \\
\pm \\
0.7\end{array}$ & $\begin{array}{l}5.0 \\
\pm \\
1.2\end{array}$ & $\begin{array}{l}0.9 \\
\pm \\
0.4\end{array}$ & $\begin{array}{l}2.5 \\
\pm \\
0.9\end{array}$ & $\begin{array}{l}2.7 \\
\pm \\
0.3\end{array}$ & $\begin{array}{l}0.16 \\
\pm \\
0.04\end{array}$ & $\begin{array}{l}0.21 \\
\pm \\
0.04\end{array}$ & $\begin{array}{l}0.76 \\
\pm \\
0.12\end{array}$ \\
\hline $\begin{array}{l}\text { Bethanechol } \\
+ \text { Captopril }\end{array}$ & 6 & $\begin{array}{c}321 \\
\pm \\
12\end{array}$ & $\begin{array}{l}2.1 \\
\pm \\
1.5\end{array}$ & $\begin{array}{l}5.6 \\
\pm \\
1.7\end{array}$ & $\begin{array}{l}8.1 \\
\pm \\
2.4\end{array}$ & $\begin{array}{l}6.4 \\
\pm \\
0.7\end{array}$ & $\begin{array}{l}6.9 \\
\pm \\
0.6\end{array}$ & $\begin{array}{l}8.6 \\
\pm \\
1.0\end{array}$ & $\begin{array}{l}0.79 \\
\pm \\
0.05\end{array}$ & $\begin{array}{l}0.68 \\
\pm \\
0.05\end{array}$ & $\begin{array}{l}1.09 \\
\pm \\
0.07\end{array}$ \\
\hline Significance- & Way Al & JOVA & & & & & & & & & \\
\hline $\begin{array}{l}\text { Bethanechol } \\
\text { Captopril } \\
\text { Interaction }\end{array}$ & & $\begin{array}{l}\text { NS } \\
\text { NS } \\
\text { NS }\end{array}$ & $\begin{array}{l}<0.01 \\
<0.01 \\
<0.05\end{array}$ & $\begin{array}{l}<0.01 \\
\text { NS } \\
\text { NS }\end{array}$ & $\begin{array}{l}<0.01 \\
\text { NS } \\
\text { NS }\end{array}$ & $\begin{array}{l}<0.01 \\
<0.05 \\
\text { NS }\end{array}$ & $\begin{array}{l}<0.01 \\
<0.05 \\
\text { NS }\end{array}$ & $\begin{array}{l}<0.01 \\
\text { NS } \\
<0.01\end{array}$ & $\begin{array}{l}<0.01 \\
\text { NS } \\
\text { NS }\end{array}$ & $\begin{array}{l}<0.01 \\
\text { NS } \\
\text { NS }\end{array}$ & $\begin{array}{l}\text { NS } \\
<0.01 \\
<0.01\end{array}$ \\
\hline
\end{tabular}

*One standard error of mean. 


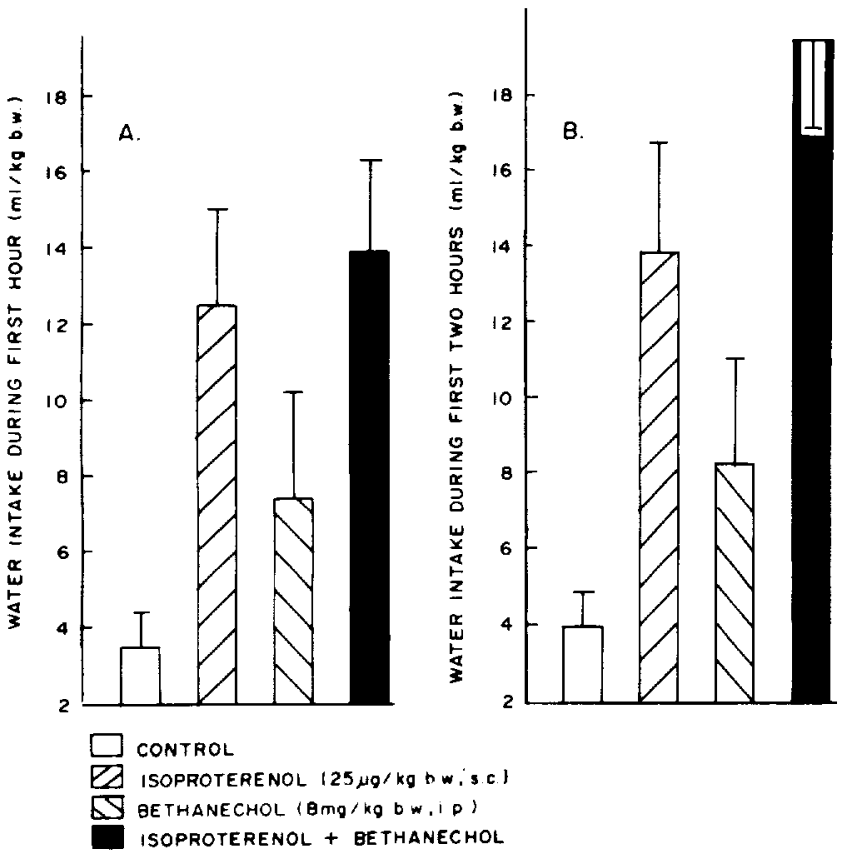

FIG. 1. Effect of administration of isoproterenol and bethanechol, alone and in combination, on water intake during the first (A) and second (B) hours after treatment. One standard error is set off at each mean.

\section{Experiment 4}

Administration of the angiotensin I converting enzyme inhibitor, captopril ( $35 \mathrm{mg} / \mathrm{kg})$, inhibited the increased water intake induced by bethanechol (Table 4). The effect of captopril was greatest during the first $\mathrm{hr}$ after treatment. Its effectiveness waned considerably during the second and third $\mathrm{hr}$ of the experiment. Captopril alone was without significant effect on water intake. The increased urine output and increased urinary sodium and potassium excretion rates induced by bethanechol were unaffected by simultaneous treatment with captopril.

\section{Experiment 5}

When isoproterenol ( $25 \mu \mathrm{g} / \mathrm{kg}, \mathrm{SC}$ ) and bethanechol ( 8 $\mathrm{mg} / \mathrm{kg}$, IP) were administered alone, both induced a significant increase in water intake during the first and second $\mathrm{hr}$ after treatment (Fig. 1). When administered together, the effects of the two treatments were additive and not interactive as determined by a two way analysis of variance.

\section{Experiment 6}

Administration of bethanechol $(8 \mathrm{mg} / \mathrm{kg}, \mathrm{IP})$ to conscious rats with an indwelling carotid cannula was accompanied by a reduction in mean blood pressure of approximately $40 \mathrm{~mm}$ $\mathrm{Hg}$ (Fig. 2A). The maximal depression occurred at approximately $10 \mathrm{~min}$ after treatment after which mean blood pressure returned toward that of controls. By 30 min after treatment, mean blood pressure of the treated group was no longer significantly different from that of the control group.

Heart rate after administration of bethanechol followed a time-course that was similar to that of mean blood pressure (Fig. 2B).
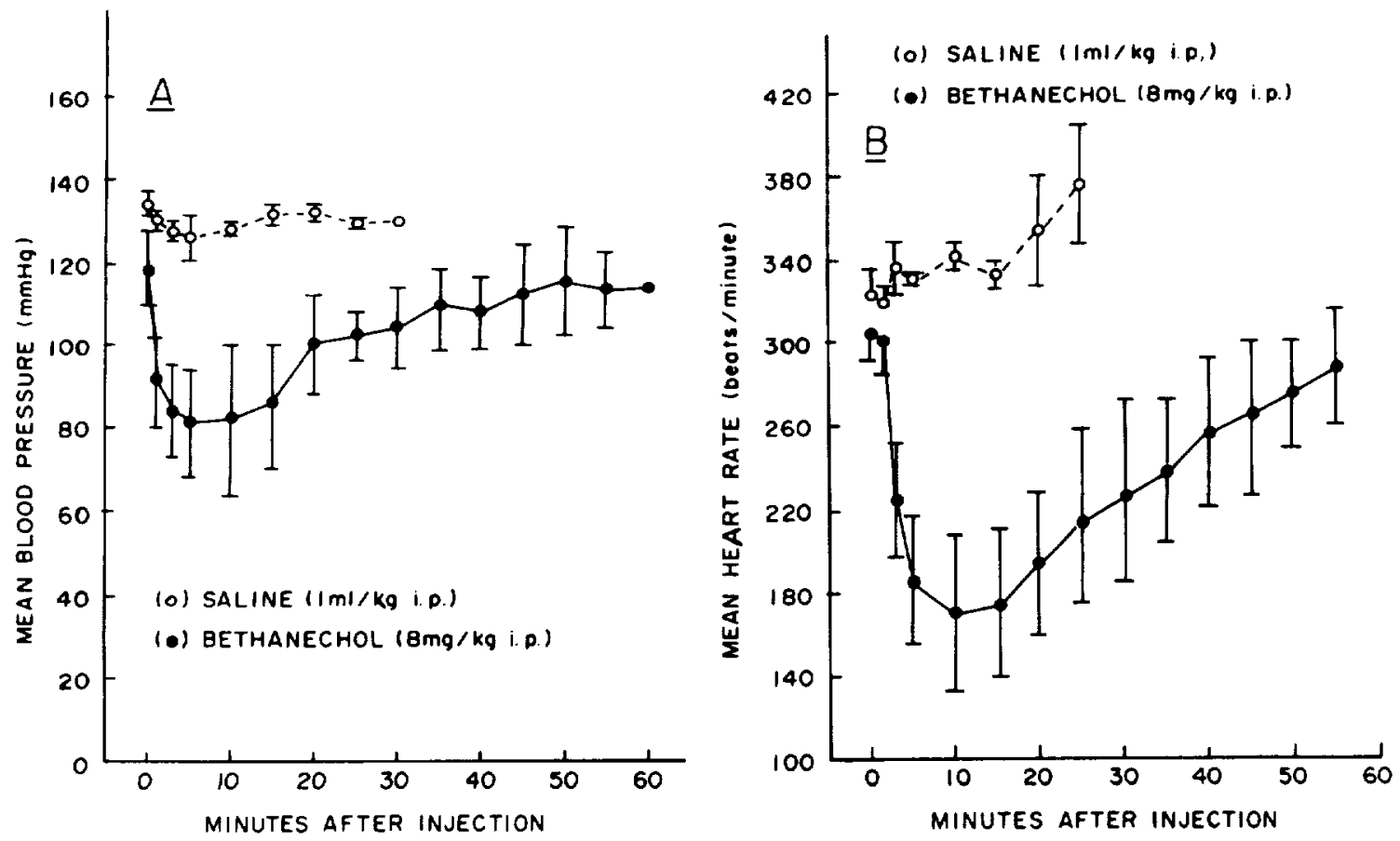

FIG. 2. Effect of administration of bethanechol on mean blood pressure (A) and heart rate (B) of conscious rats having an indwelling cannula in one carotid artery. One standard error is set off at each mean. 


\section{DISCUSSION}

Peripheral administration of graded doses of the parasympathomimetic agent, bethanechol, to rats was accompanied by a graded increase in water intake which occurred within an $\mathrm{hr}$ after treatment. The first significant effect occurred when a dose of $4 \mathrm{mg} / \mathrm{kg}$ was administered. The highest dose of bethanechol $(12 \mathrm{mg} / \mathrm{kg})$ may have induced adverse effects since this group drank considerably less water during the first hr of the study than the group administered $8 \mathrm{mg} / \mathrm{kg}$. However, the effect of the highest dose of bethanechol on water intake manifested itself during the second hr of the study when, presumably, the adverse effect had subsided.

A previous study from this laboratory using pilocarpine also revealed that it stimulated water intake when administered peripherally to rats. However, the side effects, including diarrhea, appeared subjectively to be more prevalent with pilocarpine than bethanechol. Further, Gay et al. [5] reported that drinking accompanying administration of pilocarpine to rats depended in part on the dose administered and on the time after administration that measurements were made. Maximal drinking response to graded IP doses of pilocarpine was observed at $3.75 \mathrm{mg} / \mathrm{kg}$. Doses higher that this $(7.5,15$ and $30 \mathrm{mg} / \mathrm{kg})$ progressively reduced the drinking response during the first 90 min of the study. By $180 \mathrm{~min}$ after treatment, a dose-response relationship appeared [5]. A further interesting aspect of their study was that administration of $1 \mathrm{mg}$ of either scopolamine or methyl scopolamine/ $\mathrm{kg}$ failed to affect pilocarpine-induced drinking and scopolamine may actually have enhanced it. In addition, methyl atropine $(2.0 \mathrm{mg} / \mathrm{kg})$ failed to affect pilocarpineinduced water intake. In contrast, administration of either 3.0 or $6.0 \mathrm{mg}$ atropine sulfate $/ \mathrm{kg}$ inhibited bethanecholinduced drinking. The drinking responses induced by pilocarpine and bethanechol would appear to differ in at least this important respect. Unpublished studies from this laboratory have shown that atropine sulfate $(6.0 \mathrm{mg} / \mathrm{kg}$, IP) had no effect on the water intake of rats during the course of a 3 hour experiment.

Administration of bethanechol was always accompanied by an increased urine output and urinary sodium excretion (Tables 1-4). Similar results have been reported to accompany peripheral administration of pilocarpine, as well as central administration of carbachol, to rats $[4,12]$. In contrast to the effect of peripheral administration of pilocarpine, the first significant increases in both urine output and water intake accompanying peripheral administration of bethanechol occurred at the same dose. In the case of pilocarpine, urine output increased at a lower dose than that inducing water intake [4]. Whether the effects of administration of parasympathomimetic agents on renal sodium excretion are manifested at the level of the kidney or elsewhere is unknown. While changes in renal hemodynamics resulting from the reduction in blood pressure accompanying administration of bethanechol may play a role, a reduction in glomerular filtration rate would be expected to reduce, rather than increase, renal sodium loss. Other factors are more likely to contribute to the increased renal sodium loss. In this regard it is of interest that atropine blocked the natriuretic effect of bethanechol while propranolol and captopril did not. This suggests that the natriuretic effect of bethanechol is a direct result of its parasympathomimetic activity.

The mechanism by which administration of bethanechol increases water intake appears most likely to occur by way of the renin-angiotensin system. Bethanechol may act directly at preganglionic sympathetic nerve terminals to initiate the release of catecholamines from the adrenal medulla and thereby stimulate the release of renin from the kidney. It is also likely that release of renin is reflexly initiated by the fall in blood pressure accompanying its administration (Fig. 2). The drinking response to administration of bethanechol is inhibited by concurrent treatment with either the $\beta$-adrenoreceptor antagonist, propranolol, or the angiotensin I converting enzyme inhibitor, captopril. These results also suggest that the renin-angiotensin system contributes to the bethanechol-induced water intake. Other studies have shown that both propranolol and captopril, administered alone at the doses used here, failed to affect water intake of rats $[8,9]$.

A furhter suggestion that bethanechol-induced water intake uses a pathway similar to that of isoproterenol is found in the data from Experiment 5 in which these compounds were administered singly and together (Fig. 1). The effect of simultaneous administration of the two compounds was additive rather than interactive. Thus, separate pathways for mediation of the two drinking responses are unlikely.

\section{ACKNOWLEDGEMENTS}

The authors are grateful to Mrs. Charlotte Edelstein for the illustrations and to $\mathrm{Mr}$. Thomas Connor for technical assistance.
1. Blass, E. M. and H. W. Chapman. An evaluation of the contribution of cholinergic mechanism to thirst. Physiol. Behav. 7: 679-686, 1971 .

2. Fisher, A. E. Relationships between cholinergic and other dipsogens in the central mediation of thirst. In: The Neuropsychology of Thirst: New Findings and Advances in Concepts, edited by A. N. Epstein, H. R. Kissileff and E. Stellar. Washington, DC: Winston, 1973, pp. 243-278.

3. Fisher, A. E. and J. N. Coury. Cholinergic tracing of a central neural circuit underlying the thirst drive. Scienc'e 138: 691-693, 1962.

4. Fregly, M. J. Attenuation of pilocarpine-induced drinking by chronic treatment with estrogens. Proc. Soc. exp. Biol. Med. 164: 178-183, 1980.

5. Gay, P. E., S. C. Benner and R. C. Leaf. Drinking induced by parenteral injections of pilocarpine. Pharmac. Biochem. Behav. 5: 633-638, 1976 .

6. Grossman, S. P. Eating or drinking elicited by direct adrenergic or cholinergic stimulation of hypothalamus. Science 132: 301$302,1960$.
7. Huntsberger, D. V. Elements of Statistical Inference. Boston: Allyn and Bacon, 1961, pp. 213.

8. Katovich, M. J. and M. J. Fregly. Mediation of isoproterenolinduced thirst in rats by $\beta_{2}$-adrenergic receptors. Can. $J$. Physiol. Pharmac. 56: 465-470, 1978.

9. Katovich, M. J., C. C. Barney, M. J. Fregly and R. E. McCaa. Effect of an angiotensin converting enzyme inhibitor (SQ 14,225) on $\beta$-adrenergic and angiotensin-induced thirsts. Eur. $J$. Pharmac. 56: 123-130, 1979.

10. Lazarow, A. Methods for quantitative measurement of water intake. Meth. med. Res. 6: 225-229, 1954.

11. Levitt, R. A. and R. P. Boley. Drinking elicited by injection of eserine or carbachol into rat brain. Physiol. Behav. 5: 693-695, 1970.

12. Saad, W. A., L. A. Arruda Camargo, C. R. Silva Natto. C. G. Gentil, J. Antunes-Rodrigues and M. R. Covian. Natriuresis, kaliuresis and diuresis in the rat following microinjections of carbachol into the septal area. Pharmac. Biochem. Behav. 3: 985-992, 1975 .

13. Snedecor, G. W. and W. G. Cochran. Statistical Methods, 5th edition. Ames: Iowa State University Press, 1956, pp. 237-328. 